\title{
Restructuring the Genome in Response to Adaptive Challenge: McClintock's Bold Conjecture Revisited
}

\author{
R.A. JoRGENSEN \\ Department of Plant Sciences, University of Arizona, Tucson, Arizona 85721-0036
}

\begin{abstract}
I believe there is little reason to question the presence of innate systems that are able to restructure a genome. It is now necessary to learn of these systems and to determine why many of them are quiescent and remain so over very long periods of time only to be triggered into action by forms of stress, the consequences of which vary according to the nature of the challenge to be met.
\end{abstract}

-Barbara McClintock, 1978

The prevailing view in evolutionary biology has long been that natural selection acts on genetic variants that arise by accident in a manner unrelated to adaptive challenges. Barbara McClintock's revolutionary discovery that genome structure is dynamic and responsive to conditions of stress led to a revised view incorporating the possibility that mutations can arise at widely varying frequencies, even in "bursts," in response to a variety of influences, some of which may represent adaptive challenges to the organism (McDonald 1983; Walbot and Cullis 1985). This new view of the origin of mutations retains the assumption that the mutations induced by any particular adaptive challenge are no more likely to address that challenge than any other.

McClintock, however, citing the sophistication with which organisms perceive and respond to the environment, boldly advocated a more radical position: that organisms respond to challenges by inducing mutations likely to have some adaptive value toward the particular challenge experienced. Expanding on this theme in her Nobel lecture, she challenged biologists "to determine the extent of knowledge the cell has of itself, and how it utilizes this knowledge in a 'thoughtful' manner when challenged" (McClintock 1984).

This provoked one prominent critic to suggest that McClintock's speculations "would indeed seem to verge on mysticism" and to ask (almost incredulously): "[D]oes the organism ... have foresight, conjuring up just the kind of restructuring that the occasion demands?" (Fincham 1992). It is significant, however, that McClintock placed "thoughtful" in quotation marks; never did she claim any conscious effort on the part of the plant. As Shapiro (1992) explains, McClintock often spoke in her later years of "the concept of 'smart cells', a phrase she slipped in humorously at the end of her lectures" that was used to express her deep awareness of the sophistication and complexities of cells' abilities to sense, evaluate, and respond. Thus, McClintock's phrase, "a 'thoughtful' manner," might best be interpreted as referring to $a \mathrm{com}$ - plex process that integrates information and responds according to the nature of that information.

At the time she made this conjecture, understanding of the nucleus and the genome was too limited to propose mechanisms that might underlie it. Advances in molecular biology, cell biology, and genomics over the past 25 years have shed much light on the dynamic behavior of the nucleus and its genome, making it attractive to revisit McClintock's suggestion. The purpose of any such exercise is not to show how things are, but simply to illustrate how they might be, and to help identify questions that will need to be explored experimentally before reaching any conclusions about the validity of McClintock's proposal. Here I explore how paramutation, an epigenetic gene-silencing phenomenon discovered by another pioneering maize geneticist, R. Alexander Brink, might act as both a challenge and a genomic imprinting process that could generate novel variants more likely to address this challenge than randomly generated mutations would be.

\section{CONNECTING A CHALLENGE TO A LOCUS}

Any mechanism capable of generating a biased set of mutations that are potentially adaptive in the face of the particular challenge inducing them would seem to require two principal elements: (a) information must flow preferentially to those genetic loci that are affected by the challenge, and (b) mutagenesis must occur preferentially at such loci. The suggestion has often been made that chromosomally based epigenetic states could provide a medium for guiding DNA restructuring machinery to "challenged" genes (Cullis 1987; Jablonka and Lamb 1989; Maynard Smith 1990; Monk 1990; Jorgensen 1993; Shapiro 1993). The DNA of eukaryotic genes is enveloped in chromatin, which varies in structural and functional organization and composition according to the physiological and developmental (i.e., epigenetic) information to which it has been exposed. It seems reasonable to consider that the chromatin configuration at or near a locus can influence not only gene expression, but also the accessibility of DNA restructuring enzymes to that region (Jablonka and Lamb 1995). The immune system provides an example of such a mechanism in that epigenetic modification guides the choice of one allele for rearrangement in $\mathrm{V}(\mathrm{D}) \mathrm{J}$ recombination (Rada and Ferguson-Smith 2002). 


\section{DIRECTED GENOME RESTRUCTURING IN RESPONSE TO PARAMUTATION?}

McClintock (1978) regarded sexual hybridization between taxonomically distinct species to be a prime example of the kind of "frequent accident" she thought likely to activate genome-restructuring mechanisms. Natural hybridization plays an important role in the evolution of plants through two creative outcomes: (a) the origin or transfer of new traits via introgression from one species to another and (b) the origin of entirely new species (Grant 1981; Arnold 2004). How might natural hybridization create an adaptive challenge that could trigger the production of a biased set of mutations in gene(s) whose alteration might be adaptive in the face of that particular challenge? The possibility explored here is that, in flowering plants, paramutation could constitute such a challenge by virtue of the fact that it "labels" the genes it impairs.

Paramutation is an interaction between two types of alleles, one that is "paramutagenic" and another that is "paramutable" (Brink 1960). In the presence of a paramutagenic allele, a paramutable allele is altered to become a new, "paramutant" allele, which is somatically and germinally heritable and can remain paramutant even after loss or segregation of the paramutagenic allele. Many, though not all, paramutants are metastable, exhibiting erratic expression and variable inheritance. Paramutation does not result in DNA sequence alterations, so far as is known, but is instead thought to involve changes in chromatin organization (Chandler et al. 2000). Paramutation-like interactions are not limited to alleles, but also may occur between unlinked genes, especially between unlinked transgenes as well as between transgenes and unlinked, homologous, endogenous genes, suggesting that any gene might be subject to paramutation under the right circumstances.

A favored explanation for how a paramutagenic allele (or locus) can alter a paramutable allele (or locus) invokes double-stranded RNA (dsRNA) produced by the paramutagenic gene and homologous to sequences in or adjacent to the transcriptional control elements of the paramutable gene (Finnegan and Matzke 2003). dsRNA induces changes in DNA methylation and/or chromatin organization patterns in regions of the target gene that play roles in transcription initiation.

A viable alternative explanation invokes a "homology sensing" process by which homologous sequences briefly pair and chromatin-based information is transferred from one gene to the other (Hagemann 1969; Jorgensen 1992; Matzke and Matzke 1993; Patterson et al. 1993). The existence of a homology-sensing process in somatic cells that brings homologous sequences, whether allelic or ectopic, into brief association is thought to be widespread in eukaryotes and to be capable of scanning the entire genome in somatic cells (Tartof and Henikoff 1991; Kleckner and Weiner 1993).

The hypothesis here suggests that species whose genomes are sufficiently diverged become "paramutationally incompatible" such that natural hybridization between them results in new epigenetic variants (paramu- tants) with aberrant chromatin organization at some loci, and that aberrant chromatin not only disrupts the expression of paramutant genes, but also makes them preferentially susceptible to DNA sequence rearrangements. The hypersusceptibility of such a locus would persist as long as its aberrant chromatin does, i.e., until the DNA is restructured in such a way that the new allele can be organized into "normal" chromatin. Those rearrangements that also confer an adaptive expression pattern on the new allele would survive in nature and would constitute adaptive mutations, having been produced preferentially at affected loci in response to a particular challenge.

Because plants do not sequester the germ line during early development, substantial opportunities exist for sexual transmission of somatically arising mutations and epigenetic states. Plant development subsequent to embryogenesis is based largely on apical and axillary meristems, groups of relatively undifferentiated cells that reiteratively produce vegetative structures, sometimes for many years, until they perceive a developmental or physiological signal directing them to produce reproductive structures (Walbot 1985; Klekowski 1988; Jablonka and Lamb 1995). As a consequence of this flexibility, a large developmental window (potentially lasting hundreds of years in some species) exists, during which challenges could trigger mutations in meristematic cells that will later give rise to germ cells capable of transmitting new genetic variants to sexual progeny. Somatic mutants can be subjected to selection prior to sexual transmission, and so the opportunities for somatic restructuring of genes with aberrant chromatin and incorporating selectively advantageous derivatives into the germ line are significant in plants.

\section{A ROLE FOR TRANSPOSABLE ELEMENTS IN DIRECTED GENOME RESTRUCTURING?}

The possibility that transposable elements (TEs) could play a causal role in paramutation has been discussed often (see, e.g., Krebbers et al. 1987; Martienssen 1996; Matzke et al. 1996). Here I also consider the reciprocal possibility: that certain TEs mediate rearrangements that can "repair" alleles exhibiting aberrant behavior.

Plant genomes evolve via many types of small-scale DNA sequence rearrangements, and though the linear order of genes on a chromosome tends to be conserved, individual genes are embedded in a complex and diverse matrix of sequence elements that is rapidly evolving and is largely comprised of TEs of various types (Bennetzen 2000; Feschotte et al. 2002). TE insertions are known to have created evolutionarily significant gene regulatory mutations (McDonald 1995; Kidwell and Lisch 1997), as was proposed by McClintock. TEs can also generate a wide variety of secondary rearrangements and transpositions in the locale of a TE (Lönnig and Saedler 2002), and so it is attractive to propose TEs as candidates for a DNA restructuring system that can preferentially alter aberrant loci and generate new variants.

Obviously, the appropriate cellular machinery would need to be available for restructuring the DNA sequences 
that underlie aberrant chromatin states, and yet eukaryotic TEs are normally found to be in a quiescent state, often associated with repressive chromatin. However, a variety of "stresses" are known to activate silent TEs, particularly interspecific hybridization (McClintock 1978, 1984; Wessler 1996). Interestingly, an interspecific mammalian hybrid exhibits genome-wide activation of retroelement movement and genome-wide restructuring, as well as undermethylation of DNA (O'Neill et al. 1998). In plants, newly formed polyploids often exhibit rapid, large-scale genome-wide changes in a significant fraction of sequences, up to several percent of tested sequences in just a few generations (Rieseberg 2001). Genome restructuring directed by chromatin states has been proposed to explain rapid genomic evolution in newly synthesized allopolyploids (Comai et al. 2003). A role for TEs in such restructuring has been inferred, but this still requires further investigation.

Importantly, TEs vary widely in target site specificity (Bennetzen 2000; Lönnig and Saedler 2002). Class 2 elements (DNA elements), such as McClintock's Activator $(A c)$ and Dissociation $(D S)$ elements, typically have high target specificity for genic regions in plants, including and perhaps especially the transcriptional control regions that lie $5^{\prime}$ to the transcribed regions of genes. $P$ elements in Drosophila have a strong insertional bias, with insertions generally occurring in $5^{\prime}$ gene regulatory regions at the expense of coding sequences. Although the majority of class 1 elements (retrotransposons) prefer to insert into nongenic regions, the rice element Tos 17 strongly prefers to insert into genic regions (Miyao et al. 2003). Particularly interesting is the yeast long terminal repeat (LTR) retrotransposon $T y 1$, which preferentially inserts adjacent to genes transcribed by RNA polymerase III. A hot spot for $T y 1$ insertions that occur in vivo is not a hot spot in vitro, suggesting that, in addition to a DNA sequence preference, $T y l$ also targets some aspect of chromatin organization or nuclear environment. Chromatin state also determines the target site preference of the $T y 5$ element, again suggesting that retrotransposons can recognize specific chromatin domains (Zou and Voytas 1997).

If paramutation is mediated by dsRNA molecules produced by paramutagenic genes, it is easy to see how TEs could also play a significant role in the origin of (some) paramutagenic genes. Many DNA elements tend to transpose locally and even insert at adjacent sites or within themselves, often in inverse orientation (see, e.g., Jiang and Wessler 2001). They can also create inverse repeats of sequences neighboring the insertion site, some of which cause semidominant mutations (see, e.g., Coen and Carpenter 1988). Clearly, transcriptional readthrough of such inverse repeats could result in dsRNA transcript production. In fact, TEs are known to produce dsRNA and siRNA molecules that can block gene expression (Mette et al. 2002; Sijen and Plasterk 2003; Slotkin et al. 2003). Furthermore, the effects of siRNA can spread over time to adjacent sequences (Slotkin et al. 2003).

Some inverse repeats might be expected to negatively affect the expression of adjacent or unlinked genes by dsRNA- and siRNA-mediated DNA methylation of ho- mologous sequences. These would be eliminated by natural selection, of course, whereas other siRNA-producing DNA rearrangements might be selectively neutral and so would persist until lost by mutation. When natural hybridization brings neutral variants into contact with a diverged genome, however, the possibility exists that some loci in the latter genome may have undergone independent changes that happen to make them susceptible to siRNA-producing loci in the other genome (i.e., paramutable). TEs are known to contribute sequences to promoters that are essential for normal promoter function; thus, the more divergent two populations are, the more likely a variant will arise in one that could be susceptible to a new variant in the other, which, though it may be selectively neutral in its own population, behaves as paramutagenic locus in the hybrid genotype.

Corresponding arguments could be put forward for DNA:DNA interaction-mediated paramutation in which changes in chromatin organization arise as a consequence of "ectopic pairing-like" interactions. These too could be mediated or influenced by differences in TE organization patterns in or near transcriptional control regions. Thus, the hypothesis here does not have to be limited to dsRNA-mediated mechanisms of paramutation.

\section{AN "ADAPTATION DOMAIN" FOR RESTRUCTURING THE DNA THAT UNDERLIES ABERRANT CHROMATIN?}

An intriguing further possibility is that metastable loci might reside in a replication domain distinct from the domains in which active and inactive genes are typically found, and that this domain might be preferentially targeted for DNA restructuring. In higher eukaryotes, nuclear genes are replicated and expressed in a variety of distinct temporal and functional domains (Spector 1993, 2003). In general, active genes replicate early in the cell cycle and inactive genes replicate late. Interestingly, these temporal domains can be distinguished by two distinct types of replication foci-those possessing maintenance DNA methyltransferase and those lacking it (Leonhardt et al. 1992) - and imposition of epigenetic states can require S phase and replication (Fox et al. 1997).

It is interesting to speculate that aberrant chromatin might comprise a novel "adaptation" domain, which is targeted by the molecular machinery for generating rearrangements of the DNA underlying the aberrant chromatin residing there. To illustrate the potential for such a mechanism, it is interesting to consider that in the immune system epigenetic modifications have been implicated in the choice of an allele for early replication and subsequent rearrangement (Rada and Ferguson-Smith 2002). Thus, an allele's epigenetic state can determine its replication domain, and this domain can be preferentially targeted for rearrangement. Changes in chromatin structure can even be associated with altered positioning of chromosome territories (Spector 2003). In parental imprinting in mammals, allele-specific epigenetic modifications determine differential replication timing and subnuclear localization (Gribnau et al. 2003), a basis for 
suggesting a distinct physical location for an adaptation domain within the nucleus.

Because the establishment of a new epigenetic state can require passage through $\mathrm{S}$ phase and can occur at replication foci, rearrangement of DNA underlying aberrant chromatin could even be accompanied by establishment of a new chromatin state, perhaps as the DNA leaves the replication focus. If the new chromatin state is a normal, active state, the gene would be returned to an early replicating, actively expressed domain. If not, the gene would remain in the adaptation domain and continue to be a target for DNA rearrangement.

In fragile $\mathrm{X}$ syndrome, mutant alleles with an expanded CGG repeat exhibit delayed replication timing not only locally, but also over a large region of at least $180 \mathrm{~kb}$ surrounding the repeat array (Hansen et al. 1993). This illustrates how a relatively small change in DNA sequence organization (an increase of only several hundred base pairs) can affect the replication timing of a region nearly three orders of magnitude larger. Thus, rearrangements occurring at a considerable physical distance from transcribed sequences could alter the replication domain of a gene.

\section{CONCLUSIONS}

The hypothesis presented here suggests that, in an interspecific plant hybrid, (a) some gene pairs will interact in such a way that a metastable, paramutant state arises in one or both, (b) activation of certain families of TEs is induced as another consequence of hybridization, and (c) aberrant chromatin states of paramutant genes allow preferential integration at or near these genes, as well as associated or subsequent adjacent rearrangements.

Once initiated, restructuring events would continue to arise locally as long as the chromatin remains aberrant and the restructuring machinery remains active and available. The process of "repairing" a paramutant might take several to many generations, depending on the likelihood that a new mutation alters the locus in such a way that it will move from the proposed aberrant chromatin domain to a normal chromatin domain. Natural selection, of course, will act on all new variants, favoring those that are beneficially expressed in the hybrid organism and its progeny. Obviously, recombination and segregation could also remove alleles exhibiting aberrant chromatin, but the fact that extensive, rapid restructuring does in fact occur in hybrids and allopolyploids that derive from "wide crosses" (Rieseberg 2001; Comai et al. 2003) indicates that new variants do arise quickly and abundantly enough to support such a hypothesis.

The principal objection raised to McClintock's conjecture of adaptive genome restructuring has been that it is difficult to conceive of any mechanism behind it. A counterargument suggested that natural selection ought to favor the evolution of any system able to respond more efficiently to the challenges it faces by connecting a particular challenge to the generation of a potentially "useful" spectrum of mutations to meet that challenge and so it is worthwhile looking for evidence of such sys- tems (Shapiro 1993). To bridge this gap in perceptions, it will be necessary to vastly improve our understanding of the processes underlying genome evolution by openly considering realistic molecular possibilities and developing testable hypotheses.

The hypothesis presented here will be useful if it encourages both discussion and experimental investigation of the possibilities and questions raised by it, such as whether paramutation-like events occur commonly in sexual hybrids between substantially diverged plant taxa, whether some loci exhibiting aberrant epigenetic states inhabit a distinct temporal or functional domain in the nucleus, and whether aberrant chromatin is a preferred target for certain TEs and/or other DNA restructuring systems. Also, by first addressing McClintock's conjecture in the narrow sense of paramutation, it is hoped that readers may more easily see the larger possibilities that could derive from the hypothesis, especially that of adaptive restructuring in response to environmental challenge.

\section{Beyond Paramutation}

Paramutation and transposition are generally regarded as aberrant events resulting from the breakdown of normal cellular processes and parasitism of selfish DNA elements. McClintock, on the contrary, believed that the most biologically important role of transposable elements is of a higher order, as components of sophisticated genome restructuring systems. Similarly, Brink argued that although paramutation sensu stricto is observed as an aberration, it is perhaps best viewed more broadly as a reflection of normal gene regulatory mechanisms. To explain eukaryotic gene regulation in 1960, he proposed a "paragenetic" function for chromosomes that is superimposed on their primary, genetic function of ensuring stable transmission of the genetic material. This paragenetic function would have a dual purpose: (a) to control gene expression and (b) to receive and record information about the cellular environment and transmit it mitotically throughout growth and development (Brink 1960). It is now clearly established that many gene expression states in plants that are developmentally or environmentally determined are based on chromatin that can be reprogrammed (Goodrich and Tweedie 2002), i.e., they are effectively paragenetic states as defined by Brink (Jorgensen 1994).

Later, McClintock (1967) embraced a similar view to Brink's after observing the "setting" and "erasure" of gene expression states in maize:

\footnotetext{
[A] locus is in no manner permanently modified by the events responsible for setting and erasure. It can undergo repeated cycles of this type of programming of action, ... a type of regulation of gene action that may have general significance. Action of genes could be programmed at one stage of development in a manner that would regulate their expressions at a later stage. An imposed program could be erased subsequently and the locus again readied for future programming.
}

Thus, stepping only slightly beyond the hypothesis described here, it is very tempting to speculate that environ- 
mental factors that normally modulate gene expression via paragenetic states might, under "stressful" conditions, produce aberrant chromatin states that are preferentially targeted for DNA restructuring by TE-mediated processes, thereby generating a biased spectrum of mutations potentially adaptive to the particular environmental challenge. Taking together Brink's paragenetic view of gene regulation with McClintock's systems view of transposable elements in adaptive evolution, it is not difficult to envision a sophisticated information processing system whose function is to employ aberrant paragenetic states arising under adverse circumstances to improve the evolutionary odds that an organism will discover useful genetic solutions to some of the unanticipated challenges it encounters - perhaps not a "thoughtful" process per se, but certainly an attentive one. To paraphrase McClintock (1978), it is time to explore the nature and evolutionary significance of these attentive systems for adaptive genome restructuring in response to stress, "the consequences of which vary according to the nature of the challenge to be met."

\section{ACKNOWLEDGMENTS}

The author's research in epigenetics is supported by grants from the National Science Foundation under Grant No. 9975930 in the Plant Genome Research Program and the Department of Energy's Office of Basic Energy Sciences under Grant No. DE-FG03-98ER20308. I have generally cited reviews in order to keep the list of references to a reasonable length, and so I must apologize to those whose important primary research has not been cited here. Readers may access the primary literature via these reviews.

\section{REFERENCES}

Arnold M.L. 2004. Transfer and origin of adaptations through natural hybridization: Were Anderson and Stebbins right? Plant Cell 16: 562.

Bennetzen J.L. 2000. Transposable element contributions to plant gene and genome evolution. Plant Mol. Biol. 42: 251.

Brink R.A. 1960. Paramutation and chromosome organization. Q. Rev. Biol. 35: 120.

Chandler V.L., Eggleston W.B., and Dorweiler J.E. 2000. Paramutation in maize. Plant Mol. Biol. 43: 121.

Coen E.S. and Carpenter R. 1988. A semi-dominant allele, niv525 , acts in trans to inhibit expression of its wild-type homologue in Antirrhinum majus. EMBO J. 7: 877.

Comai L., Madlung A., Josefsson C., and Tyagi A. 2003. Do the different parental 'heteromes' cause genomic shock in newly formed allopolyploids? Philos. Trans. R. Soc. Lond. B Biol. Sci. 358: 1149.

Cullis C.A. 1987. The generation of somatic and heritable variation in response to stress. Am. Nat. (suppl.) 103: S62.

Feshotte C., Jiang N., and Wessler S.R. 2002. Plant transposable elements: Where genetics meets genomics. Nat. Rev. Genet. 3: 329 .

Fincham J.R.S. 1992. Book review: "The dynamic genome: Barbara McClintock's ideas in the century of genetics." Nature 358: 631 .

Finnegan E.J. and Matzke M.A. 2003. The small RNA world. $J$. Cell Sci. 116: 4689.

Fox C.A., Ehrenhofer-Murray A.E., Loo S., and Rine J. 1997. The origin recognition complex, SIRI, and the S phase requirement for silencing. Science 276: 1547.
Goodrich J. and Tweedie S. 2002. Remembrance of things past: Chromatin remodeling in plant development. Annu. Rev. Cell Dev. Biol. 18: 707.

Grant V. 1981. The species situation in plants. In Plant speciation, 2nd edition, p. 70. Columbia University Press, New York, New York.

Gribnau J., Hochedlinger K., Hata K., Li E., and Jaenisch R. 2003. Asynchronous replication timing of imprinted loci is independent of DNA methylation, but consistent with differential subnuclear localization. Genes Dev. 17: 759.

Hagemann R. 1969. Somatic conversion (paramutation) at the Sulfurea locus of Lycopersicon esculentum Mill. III. Studies with trisomics. Can. J. Genet. Cytol. 11: 346.

Hansen R.S., Canfield T.K., Lamb M.M., Gartler S.M., and Laird C.D. 1993. Association of Fragile X Syndrome with delayed replication of the FMR1 gene. Cell 73: 1403.

Jablonka E. and Lamb M.J. 1989. The inheritance of acquired epigenetic variations. J. Theor. Biol. 139: 69.

. 1995. Epigenetic inheritance and evolution. Oxford University Press, Oxford, United Kingdom.

Jiang N. and Wessler S.R. 2001. Insertion preference of maize and rice miniature inverted repeat transposable elements as revealed by the analysis of nested elements. Plant Cell 13: 2553.

Jorgensen R. 1992. Silencing of plant genes by homologous transgenes. Agbiotech News Info. 4: 265N.

- 1993. The germinal inheritance of epigenetic information in plants. Philos. Trans. R. Soc. Lond. B Biol. Sci. 339: 173.

. 1994. Developmental significance of epigenetic impositions on the plant genome: A paragenetic function for chromosomes. Dev. Genet. 15: 523.

Kidwell M.G. and Lisch D. 1997. Transposable elements as sources of variation in animals and plants. Proc. Natl. Acad. Sci. 94: 7704.

Kleckner N. and Weiner B.M. 1993. Potential advantages of unstable interactions for pairing of chromosomes in meiotic, somatic, and premeiotic cells. Cold Spring Harbor Symp. Quant. Biol. 58: 553.

Klekowski E.J., Jr. 1988. Mutation, developmental selection, and plant evolution. Columbia University Press, New York.

Krebbers E., Hehl R., Piotrowiak R., Lönnig W.E., Sommer H., and Saedler H. 1987. Molecular analysis of paramutant plants of Antirrhinum majus and the involvement of transposable elements. Mol. Gen. Genet. 209: 499.

Leonhardt H., Page A.W., Weier H., and Bestor T.H. 1992. A targeting sequence directs DNA methyltransferase to sites of DNA replication in mammalian nuclei. Cell 69: 865.

Lönnig W.-E. and Saedler H. 2002. Chromosome rearrangements and transposable elements. Annu. Rev. Genet. 36: 389.

Martienssen R. 1996. Epigenetic phenomena: Paramutation and gene silencing in plants. Curr. Biol. 6: 810 .

Matzke M.A. and Matzke A.J.M. 1993. Genomic imprinting in plants: Parental effects and trans-inactivation phenomena. Annu. Rev. Plant Physiol. Plant Mol. Biol. 44: 53.

Matzke M.A., Matzke A.J.M., and Eggleston W.B. 1996. Paramutation and transgene silencing: A common response to invasive DNA? Trends Plant Sci. 1: 382.

Maynard Smith J. 1990. Models of a dual inheritance system. $J$. Theor. Biol. 143: 41.

McClintock B. 1967. Genetic systems regulating gene expression during development. Dev. Biol. Suppl. 1: 84.

- 1978. Mechanisms that rapidly reorganize the genome. Stadler Genet. Symp. 10: 25.

. 1984. The significance of responses of the genome to challenge. Science 226: 792.

McDonald J.F. 1983. The molecular basis of adaptation: A critical review of relevant ideas and observations. Annu. Rev. Ecol. Syst. 14: 77.

. 1995. Transposable elements: Possible catalysts of organismic evolution. Trends Ecol. Evol. 10: 123.

Mette M.F., van der Winden J., Matzke M., and Matzke A.J.M. 2002. Short RNAs can identify new candidate transposable 
element families in Arabidopsis. Plant Physiol. 130: 6.

Miyao A., Tanaka K., Murata K., Sawaki H., Takeda S., Abe K., Shinozuka Y., Onosato K., and Hirochika H. 2003. Target site specificity of the Tos 17 retrotransposon shows a preference for insertion within genes and against insertion in retrotransposon-rich regions of the genome. Plant Cell 15: 1771.

Monk M. 1990. Variation in epigenetic inheritance. Trends Genet. 6: 110.

O’Neill B.J., O’Neill M.J., and Graves J.A. 1998. Undermethylation associated with retroelement activation and chromosome remodelling in an interspecific mammalian hybrid. $\mathrm{Na}$ ture 393: 68.

Patterson G.I., Thorpe C.J., and Chandler V.L. 1993. Paramutation, an allelic interaction, is associated with a stable and heritable reduction of transcription of the maize $b$ regulatory gene. Genetics 135: 881 .

Rada R. and Ferguson-Smith A.C. 2002. Epigenetics: Monoallelic expression in the immune system. Curr. Biol. 12: R108.

Rieseberg L.H. 2001. Polyploid evolution: Keeping the peace at genomic reunions. Curr. Biol. 11: R925.

Shapiro J.A. 1992. Barbara McClintock, 1902-1992. Bioessays 14: 791.
1993. Genome organization, natural genetic engineering, and adaptive mutation. Trends Genet. 13: 98.

Sijen T. and Plasterk R.H.A. 2003. Transposon silencing in the Caenorhabditis elegans germ line by natural RNAi. Nature 426: 310 .

Slotkin R.K., Freeling M., and Lisch D. 2003. Mu killer causes the heritable inactivation of the Mutator family of transposable elements in Zea mays. Genetics 165: 781.

Spector D.L. 1993. Macromolecular domains within the cell nucleus. Annu. Rev. Cell Biol. 9: 265.

- 2003. The dynamics of chromosome organization and gene regulation. Annu. Rev. Biochem. 72: 573.

Tartof K.D. and Henikoff S. 1991. Trans-sensing effects from Drosophila to humans. Cell 65: 201.

Walbot V. 1985. On the life strategies of plants and animals. Trends Genet. 1: 165.

Walbot V. and Cullis C.A. 1985. Rapid genomic change in higher plants. Annu. Rev. Plant Physiol. 36: 367.

Wessler S.R. 1996. Plant retrotransposons: Turned on by stress. Curr. Biol. 6: 959.

Zou S. and Voytas D.F. 1997. Silent chromatin determines the target preference of the Saccharomyces retrotransposon Ty5. Proc. Natl. Acad. Sci. 94: 7412. 


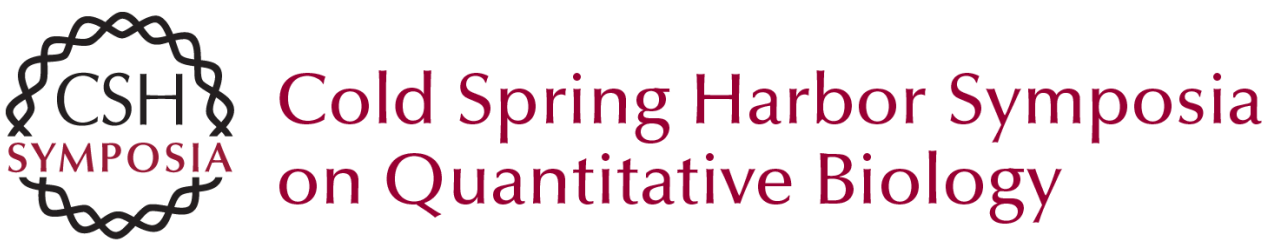

\title{
Restructuring the Genome in Response to Adaptive Challenge: McClintock's Bold Conjecture Revisited
}

\author{
R.A. JORGENSEN
}

Cold Spring Harb Symp Quant Biol 2004 69: 349-354

Access the most recent version at doi:10.1101/sqb.2004.69.349

References This article cites 52 articles, 13 of which can be accessed free at: http://symposium.cshlp.org/content/69/349.full.html\#ref-list-1

\section{License}

Email Alerting

Receive free email alerts when new articles cite this article - sign up in Service the box at the top right corner of the article or click here. 